\title{
The Use of Artificial Intelligence in Industry 4.0
}

\author{
Maksim Sharabov ${ }^{1}$, Georgi Tsochev ${ }^{2}$ \\ Author addresses: \\ ${ }^{1}$ Technical University of Sofia, Faculty of Electronic Engineering and Technologies, Sofia, Bulgaria. \\ ${ }^{2}$ Technical University of Sofia, Faculty of Computer Systems and Control, Sofia, Bulgaria \\ Emails:msharabov@tu-sofia.bg,gtsochev@tu-sofia.bg
}

\begin{abstract}
This article presents a brief overview of the effect of new technologies, how they are changing the manufacturing process, and how the machines are starting to get a lot smarter thanks to the artificial intelligence. The focus is over the examination of Industry 4.0 and how it revolutionized the whole manufacturing segment and what promise of a better, more efficient future it brings. This analysis focuses primarily on how artificial intelligence is integrated, what benefits it brings, and how big of an improvement it is over basic programming. Part of the research is based on 771 publications tracked over the past three to five years. Publications are within some of the well-known databases Scopus, Web of Science, and IEEE. We will examine the basic use case scenarios where AI is crucially needed and how a new generation of the factory can look and feel like a living human being.
\end{abstract}

Keywords: Industry 4.0, artificial intelligence, predictive analytics, predictive maintenance, industrial robotics, computer vision.

\section{Introduction}

The most significant transformation related to the way products are manufactured is the digitization. The fourth industrial revolution, known as Industry 4.0 aims to optimize third computerized industrial revolution (Industry 3.0). This requires developing of smart equipment with access to more data thus becoming more efficient and productive by making real-time decisions [1], [2]. The fourth industrial revolution significantly based on digital transformation and contributes to its sustainability. A review of the fundamental design principles and technology trends of Industry 4.0 and the architectural design of Industry 4.0 is presented in [3].

The Industry 4.0 put the focus on the following innovations: 
- Cyber-physical system (CPS) - mechanical device manipulated by computer-based algorithms,

- The Internet of things (IoT) - interconnected networks of devices embedded with computerized sensing, scanning and monitoring capabilities,

- Cloud computing - offsite network hosting and data backup,

- Cognitive computing - technological platforms based on artificial intelligence.

It is found, that critical thinking, problem-solving, communication and teamwork have a significant impact on the development of innovations, which are the basis in the era of artificial intelligence [4]. Industry 4.0 relies on the Internet of Services to create modular manufacturing opportunities as the system components are designed to integrate and to realize personalized scenarios where the customer could control the design of final product [5], [6]. This is due to the technological advancements in the areas of artificial intelligence, cloud computing and smart devices. The successful integration of new technology relies on well-designed systems based on principles:

- interoperability,

- virtualisation,

- decentralisation,

- real-time capability,

- service orientation, and

- modularity.

Interoperability makes possible different systems, devices or applications to communicate each other without involving the end user. Virtualization allows the CPS to monitor physical processes using sensors, obtained data are used to build virtual and simulation models by providing a virtual copy of the physical world. The decentralization principle means that CPS should make decisions on their own to perform the tasks as autonomously as possible [7]. Real-time capability principle demands to collect and analyse the data in real time to provide the ability to react in case of failure and in-time making the proper decisions. The key design principle of Industry 4.0 is the service orientation. It enables the services of companies, cyberphysical systems, and humans to be made available and also enables new types of services to be created. In most cases this is realize by service-oriented architecture where the software services carry out small functions. Last but not least is the modular design. The design of modular systems enables flexible adaptation to the changing user requirements by replacing of some modules [8]. The modular systems can be adjusted easily in case of changing some functions or product characteristics. Using of the standardized software and hardware interfaces the new modules could be automatically identified and utilized via Internet of Services (IoS). 
All of these could be seen in our daily life by extensively use of digital technologies. The basic engine of the digitalization is the artificial intelligence (AI). Today much more manufacturing systems rely on industrial AI [9], [10] Fig. 1.

The development of artificial intelligence (AI) during the years is shown in

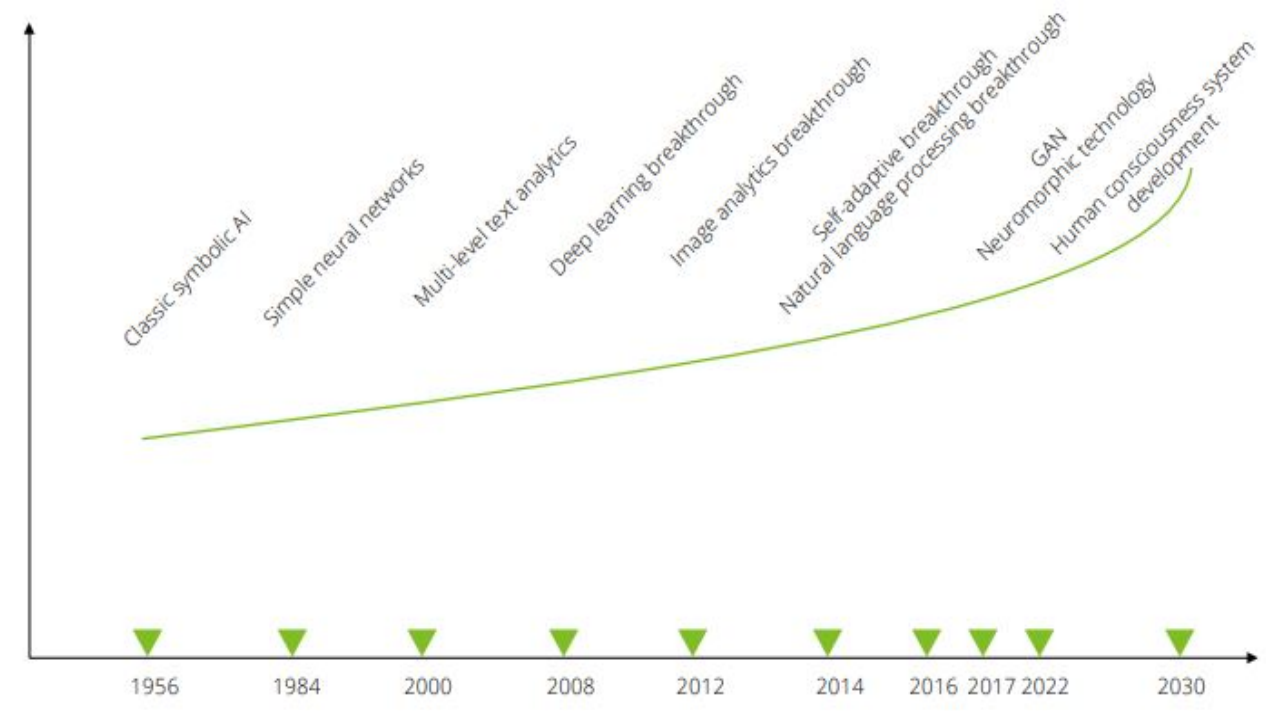

Fig. 1. History of AI development [28]

Our analysis is based on the components mentioned earlier and from there will be derived how those systems use AI. Let us see what AI is first. It tries to simulate human intelligence and machine are programmed to think like human. The basic premise is to achieve a new way for machines to perceive the world around them. The motive is that they exhibit traits associated with a human mind - learning and problem-solving.

Cyber-physical systems are managed by computer generated algorithms. Those systems rely on close integration between hardware and software more than ever and can adjust to the current situation - a very good example is the autopilot system and robotic production. The latter is different than the ordinary automation, as here all devices are connected to the network and they communicate to each other to adjust the workflow and the potential in autonomy, efficiency, functionality, and safety.

$I o T$ - it is a system of connected computer devices, mechanical and digital and individual people and even animals. They are given unique indicators, which have some internet protocol (address) and they send information in the network so that the workflow continues to be efficient.

With the "forth industrial revolution" is introduced the cloud computing. The end user thanks to the new generation of networks, he has access to powerful servers 
with a lot of calculating power and/or a lot of space for information which the local machine cannot provide and can be accessed from every part of the world anytime.

Cognitive computing directly introduces the use of AI. It has the task to simulate human decisions and thinking. So one program when is executed usually gives only one result, but there are cases when can be set of results and the next one in chain can use on one, so a human decides which one to give the program in line. The idea here is that not always human is available so AI can be used to pick the right result so that the flow can continue. Other benefit is that this can be a lot faster, efficient, and reliable, because a human can never be that fast and/or that precise, so the potential here is big, but is quite changeling. To have this kind of result and AI must have a lot of data to train from and a lot of computing power to do it efficiently.

\section{Research Methodology}

The analysis of the use of AI in Industry 4.0 adequately reflects the changes taking place in the last 3-4 years in the field of the industrial revolution and the mass penetration of AI. Artificial intelligence offers great opportunities for industry. It can make it more efficient, more flexible and more reliable.

The industry is becoming increasingly digital. The data flowing through it is getting bigger. Their processing and analysis is becoming more complex. The volumes of data in the production environment are the basis on which digital images of entire installations and systems are generated. The good news is that this can already be achieved step-by-step using AI. The main subject areas that utilize AI are summarized in Fig. 2.

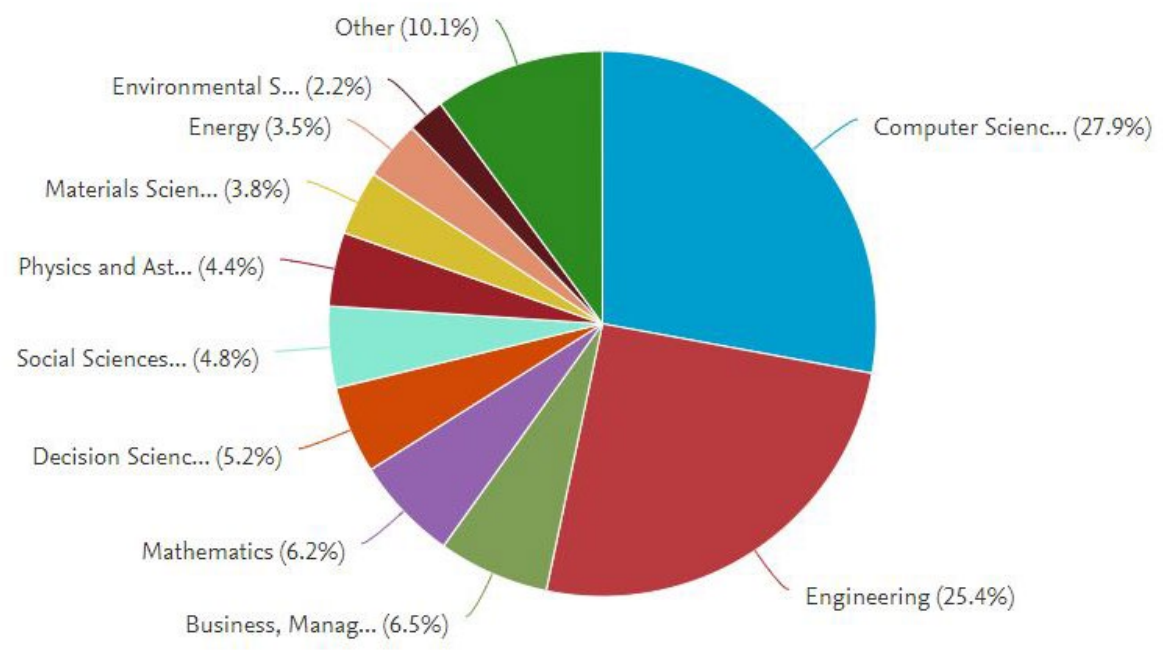

Fig. 2. Main subject areas [11], [12] 
The study is part of a project to study the security of cyber-physical connections in the context of computer networks using next-generation methods, this article provides a basis and beginning of the essential work to create a conceptual model for the protection of industrial systems. This model will be based on artificial intelligence methods suitable for IDP/IPS protection. The aim was to get an overview of AI methods from around the world, to understand the means of operation and the potential impact on business or industries. The study is based on scientific, scientificapplied and applied publications tracked over the past three to five years. There are 771 publications covering the period of years 2015 - 2020 [11], [12]. Analysed documents were subdivided in types as follows: scientific papers -666 , articles in press $-32.6 \%$, books $-0.5 \%$, book chapters $-3.5 \%$, conference papers $-55.4 \%$, conference reviews $-1.8 \%$, editorial documents $-0.4 \%$, letters $-0.1 \%$, reviews $5.2 \%$, others $-0.1 \%$

\section{AI in Industry 4.0}

Now that we understand the basics of this revolution, let us see how the AI founds itself in his place and to see how important is. Its use can be seen in few very fundamental examples for the industry as:

- Predictive Analytics,

- Predictive Maintenance,

- Industrial Robotics,

- Computer Vision,

- Inventory management.

The top 10 of the industries that benefit from AI are illustrated in Fig. 3.

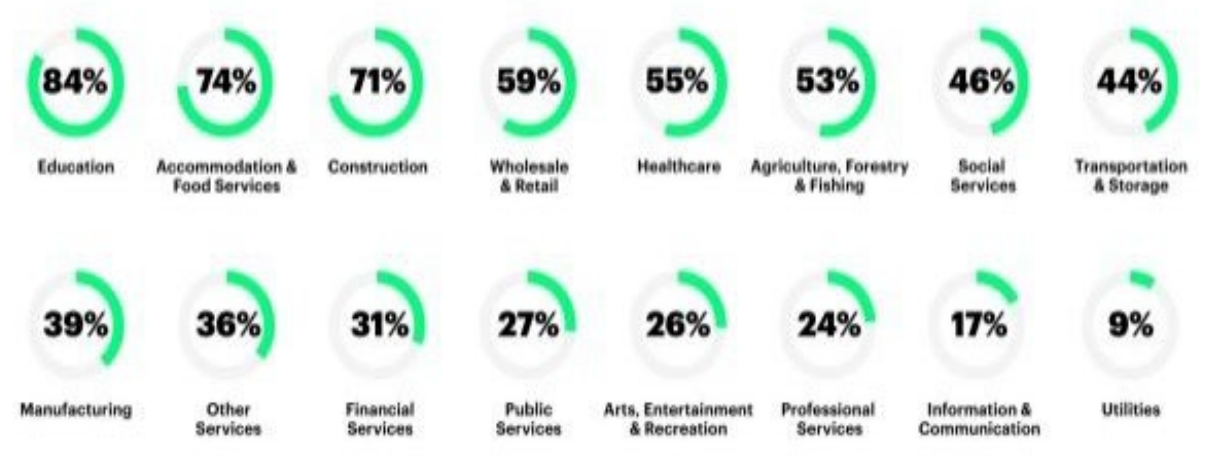

Fig. 3. Top 10 industries benefiting by AI

\subsection{Predictive analytics}

Predictive Analytics has a base fundamental idea, to work through a lot of data before, during and after the process. This can be achieved by use of AI. It can do this 
kind of computing and output to mathematical model and from there it can be used to generate different patterns that give information about what could happen based on what has happened before. The workflow for it is quite step by step. First you need data, a lot data, from files, databases, sensors etc., then data is being pre-processed. In this current step the data is messy, so it needs to be reduced and transformed, when that is done, features from it are extracted. The next step is to develop a predictive model. This is achieved by using machine learning to generate a model, the parameters in it need to be optimized so the error rate is kept to a minimal level and when that is done, the model needs to be validated through various tests and simulations. The final step is to integrate those analytics with system in the whole workflow witch the predictive analytics took place. This is done by integrating it with desktop apps, enterprise scale systems like MATLAB, Excel, Java, C/C++, .exe, .NET, .dll, Python, and embedded devices and hardware. This is the very basic concept, now let us dive deeper.

The term predictive analytics means predictive modelling, which model the data with different methods. There are three main models [13]:

- Predictive models,

- Descriptive models,

- Decision models.

The predictive models are used to study the connection between a few samples of the examined unit and could involve conditions under uncertainty [14]. They are usually used in fraud detection models, also the calculations are typically performed during live transactions, typically, to evaluate the risk opportunity of a given customer or transaction. Some of the predictive models could integrate optimization techniques to determine the proper decisions [15]. The descriptive models measure the relation in data and is used to classify it in groups. The difference between the first two are clarified in [8]. The last model is the decision model. It describes the relationship between all the segments of the known data, including the results from predictive models. A comparison of the features of Industry 4.0 against quality models and some recommendations for future quality models are proposed in [16].

The techniques used in predictive analytics are categorised into regression and machine learning techniques. The core of predictive analytics is the regression techniques (models). The key is on creating a mathematical equation as a representation of the interactions between the different variables in consideration. The models that can be used while performing predictive analytics are [17]:

- Linear regression model,

- Discrete choice models,

- Logistic regression,

- Probit regression,

- Multinomial logistic regression,

- Logit versus probit,

- Time series models, 
- Survival or duration analysis,

- Classification and regression trees (CART),

- Multivariate adaptive regression splines.

\subsection{Predictive maintenance}

Predictive maintenance, some may consider it to be part of the predictive analytics, yet they may sound like doing almost the same thing, but they differ in one key area, how they use the collected data [15]. The analytics can be used for the whole workflow (Fig. 3), but the maintenance only gives an assessment when manufacturing equipment can defect, of course, the data for that is collected from the whole manufacturing process.

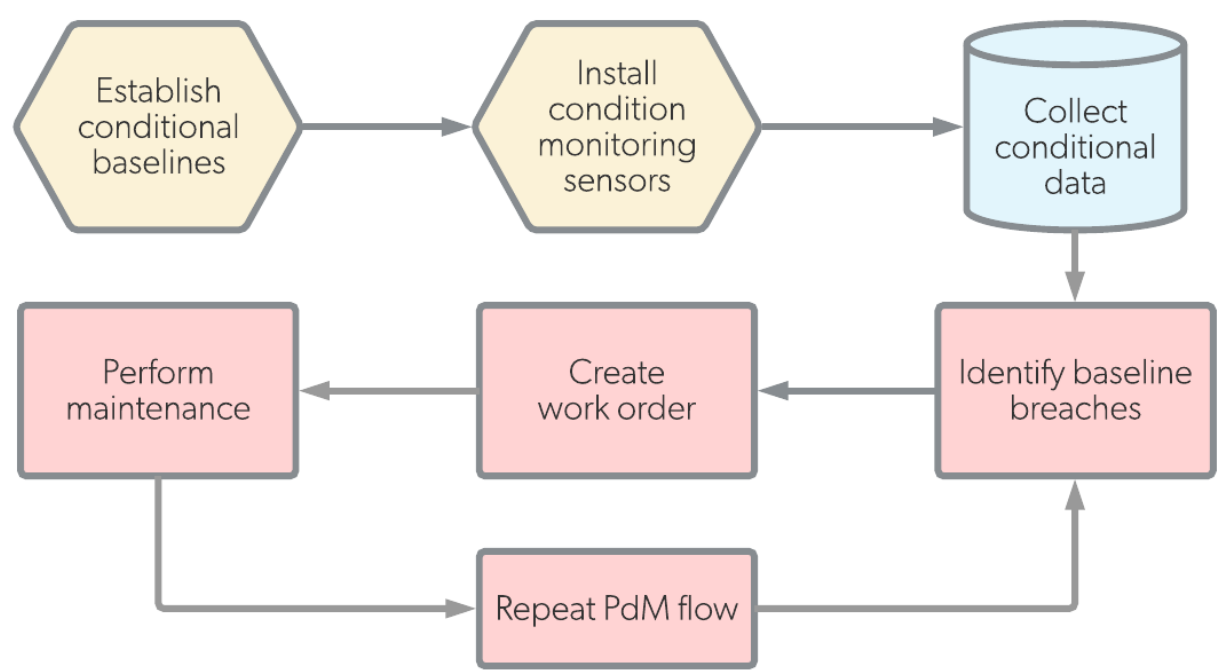

Fig. 3 Predictive maintenance workflow example [31]

We will look at how machine learning is used in predictive maintenance. First, there are needed three models so that it can be effective - having the right data available, framing the problem appropriately, and evaluating the predictions properly.

First, we need to build a failure model; to do that is required enough historical data that allows us to capture information about events leading to failure. Another key component is the mechanical properties, average use, and operating conditions. When collecting data for creating a failure model one must consider:

- What types of failure can occur?

- How the failure process looks like?

- Which parts of the system could be related to each type of failure?

When framing a problem, there are few methodical questions that must be answered. The whole process of that is explained in [18], but in general, we need to know what kind of result we want, how long in advance should the model be able to 
indicate that a failure will occur and what is the performance target. There are many strategies used in machine learning. They all have their pros and cons, because not every strategy can be used everywhere that must be considered when some wants to integrate predictive maintenance. There are two ways for approaching:

- Classification approach,

- Regression approach.

The data types used for predictive maintenance are shown in Fig. 5.

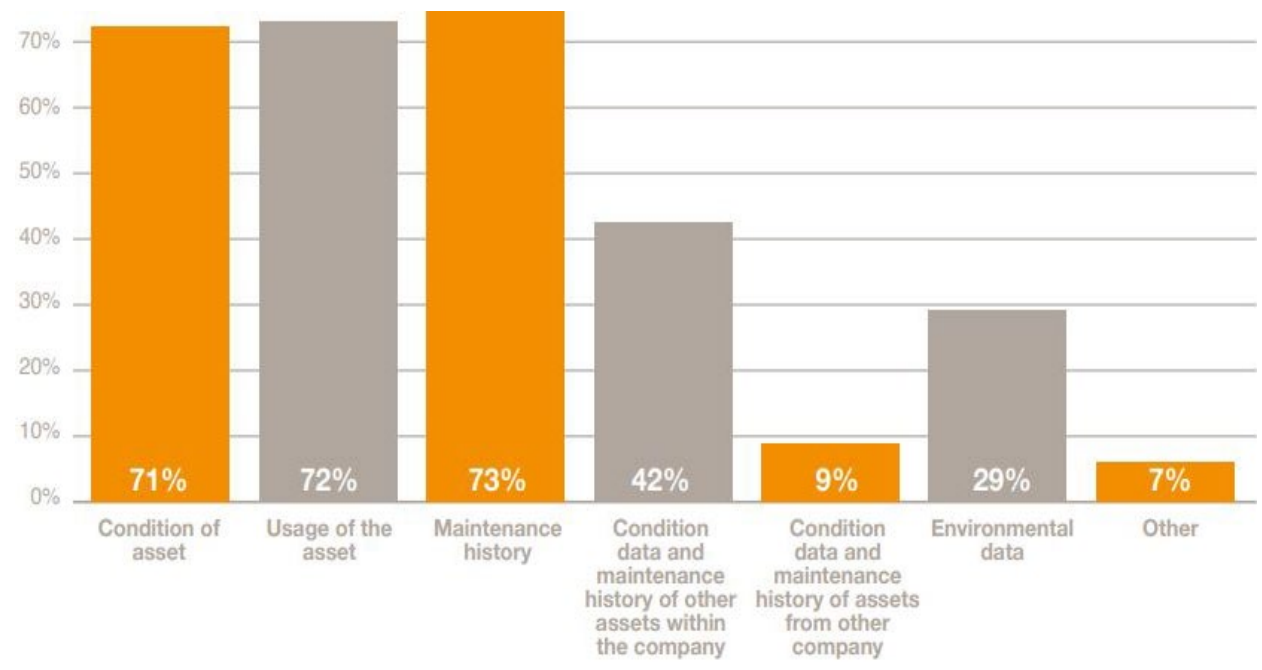

Fig. 5 Types of data are used for predictive maintenance [29]

The former approach only provides a Boolean answer, but can provide greater accuracy with less data. The latter needs more data although it provides more information about when the failure will happen. In the article [19] are explored both approaches using the NASA engine failure dataset.

Two additional strategies for predictive maintenance are mentioned in [18]: 1) flagging anomalous behaviour and 2) survival models for the prediction of failure probability over time. They are explained in detail but the bottom line is the former is often used when a failure is not allowed or there is no failure and the latter is when you are interested in the degradation process itself.

\subsection{Industrial Robotics}

Industrial robotics with AI gives way to a new generation of joint robots [20]. They are designed to work with people on the assembly line. This gives them the opportunity to be implemented a lot faster thanks to their smarter design. Industrial robotics and predictive maintenance are going hand to hand actually. Thanks to this new technology a robot can monitor their own accuracy and performance, signalling when maintenance is required to avoid expensive downtime. The AI allows also a 
new way a robot to be programmed, for example -a particular task is presented, then the robot attempts to do it with varying level of success, it learns from his mistakes, until it can perform it successfully with a high degree of repeatability.

The additional benefits are related to increased operating and reduced programming time and higher efficiency using some intuitionistic fuzzy techniques to control robot motion [21] or by using speech applications for human-robot interaction systems [22].

Some of the key benefits of the industrial robotics power by the AI include [23]:

- Vision System - improved depth and image recognition thanks to the integration of machine learning, semantic segmentation, and scene understanding;

- Scalability - their machine vision thanks to the deep learning, a 3D CAD model is no longer required and the artificial neural network can automatically identify the object in the image after training;

- Intelligent placement.

\subsection{Computer Vision}

Computer vision is a fundamental type of AI. We all have experienced it, even without our knowing. Basically it replicates the complexity of the human vision and enables computers to identify and process objects in images like human would. Thanks to neural networks and AI, the field evolved in the last few years and has been able to surpass humans in some tasks related to detecting and labelling objects. This is all very thoroughly explained in [24], but in general the driving force is the amount of data we use to train the vision. The method of computer is vision is quite simple actually, the image is broken into individual pixels and is trying to make sense. Although it sounds simple there is a bigger picture of all this. It is a complicated process when an information is interpreted from pixels.

The basic concept of interpretation is explained in details at [24], [25]. Machine learning is in the core of computer vision and is integrated very deeply in manufacturing [26] and things we use every day. This is all achieved they program "features" that could detect specific patterns in images. To achieve it they use fundamental algorithms like - logistic and linear regression, decision trees. Their purpose is to classify images and detect objects in them. This can have very wide use scenarios from the camera in our pocket for better pictures to using it in manufacturing for identifying defects in products.

In contrast, let us look when AI was not on the table. The engineers used to take much more time in developing, because they could not train the machines and had to do a lot of manual coding to achieve even remotely accurate results. This methodology had three main steps:

- Create a database,

- Annotate images, 
- Capture new images.

These main steps are explained in [24]. In a few words, there was a lot of manual work and thanks to machine learning, the process has become dramatically more efficient and easy.

In the past supercomputers were not up to the task to do this kind of stuff efficiently (sometimes they took even weeks at a time to process something like that), but thanks to new generation of hardware and software this can be done in matter of seconds, provided you have the power or a good internet connection. Yes, internet connection. In most cases actually this kind of computing is done on the cloud, thanks to their much more powerful hardware. This is all achieved because tech giants agreed to share their machines with the world for machine learning work.

There a lot of use cases for computer vision such us [24]:

- Self-Driving Cars,

- Facial Recognition,

- Augmented and Mixed Reality,

- Healthcare.

Of course, there are still challenges. There is still room for improvement, but we hit another wall. To have near perfect like eye vision we still need to understand how it works. Scientists have a lot of way to go before understanding the eyes completely and how they communicate with the brain.

The workflow of computer vision can be summarised in these few things that it wants to find in a photograph (yes, the machine takes a photograph and from there the whole analysis starts): Object Classification; Identification; Verification; Detection; Segmentation; and Recognition.

\subsection{Inventory management}

Not the most interesting use of AI, but still just as important - inventory management. If the inventory is managed perfectly this would optimise the workflow quite dramatically, this is all achieved through AI, thanks to its computing power and the ability to work with databases like no other human (in terms of speed and reliability). When it is integrated correctly it can provide information when to order supplies or not. This can tell when to invest or not thanks to the data it has collected and the machine learning to process it. This is done like predictive analytics but here it purely concentrated on managing the inventory. It looks for patterns in previous years and gives results based on the data it has collected. You may think that this is boring or useless, but in the world 1.1 trillion dollars are tied in inventory and maybe in the future thanks to AI we may finally use them.

The basic things of AI help with [27]:

- Automated Inventory Monitoring,

- Data Mining,

- Robot Automation,

- Reducing Forecasting Errors and Improving Customer Satisfaction. 


\section{Conclusion}

The artificial intelligence has a major impact on Industry 4.0. In this respect, the current article reviews some applications that benefit from AI. From the examined sources is could be concluded that the heart of nowadays industry is the AI (most commonly the machine learning), every aspect is connected to it and they all share that. Also the new way of the industry could be interpreted that it is like human with brain, eyes and physical touch to the world. In the end, everything we try to do or revolutionise is in our image. There is nothing more perfect than the human way of interaction, we try to build that, just on bigger scale, that's industry 4.0.

As part of a project to study the security of industrial systems in the context of computer networks using next-generation methods, this article provides a basis and beginning of the essential work to create a conceptual model for their protection.

\section{Acknowledgment}

This research is realized and funded under the scientific research project No 201ПР0010-09 "Investigating the security of cyber-physical connections in the context of computer networks through new generation methods" by the contract with TU-Sofia Internal Competition-2020.

\section{References}

1 Souza, M. L. H., C. Andre da Costa, G. de Oliveira Ramos, Rodrigo da Rosa Righi. A survey on decision-making based on system reliability in the context of Industry 4.0. Journal of Manufacturing Systems 56, 133-156 (2020).

2 Borissova, D., Mustakerov, I.: An integrated framework of designing a decision support system for engineering predictive maintenance. Int. Journal Information Technologies \& Knowledge 6(4), 366-376 (2012).

3 Ghobakhloo, M.: Industry 4.0, digitization, and opportunities for sustainability. Journal of Cleaner Production 252, 119869 (2020).

4 Rampersad, G.: Robot will take your job: Innovation for an era of artificial intelligence. Journal of Business Research 116, 68-74 (2020).

5 Mustakerov, I., Borissova, D.: Data structures and algorithms of intelligent Webbased system for modular design. International Journal of Computer Science and Engineering 7(7), 87-92 (2013).

6 Mustakerov, I., Borissova, D.: An intelligent approach for optimum maintenance strategy defining. In: INISTA, IEEE International Symposium on., DOI: 10.1109/INISTA.2013.6577666 (2013).

7 Bousdekis, A., Apostolou, D., Mentzas, G.: A human cyber physical system framework for operator 4.0 - artificial intelligence symbiosis. Manufacturing Letters 25, 10-15 (2020).

8 Borissova, D., Keremedchiev, D.: Product configuration design via group decision making and combinatorial optimization. Comptes rendus de l'Académie bulgare des Sciences 72(9), 1251-1261 (2019). 
9 Lee, J., Davari, H., Singh, J., Pandhare, V.: Industrial Artificial Intelligence for industry 4.0-based manufacturing systems. Manufacturing Letters 18, 20-23 (2018).

10 Merayo, D., Rodriguez-Prieto, A., Camacho, A.M.: Comparative analysis of artificial intelligence techniques for material selection applied to manufacturing in Industry 4.0. Procedia Manufacturing 41, 42-49 (2019).

11 Scopus, https://www.scopus.com/ [Link accessed June 20 2020].

12 Web of Science, https://apps.webofknowledge.com [Link accessed June 20 2020]

13 Non-Linear Predictive Model, https://www.gabormelli.com/RKB/NonLinear_Predictive_Model [Link accessible as June 20 2020].

14 Borissova, D., Mustakerov, I., Grigorova, V.: Engineering systems maintenance by optimal decision making strategies under uncertainty conditions. Problems of Engineering Cybernetics and Robotics 63, 14-21 (2011).

15 Borissova, D., Mustakerov, I.: A concept of intelligent e-maintenance decision making system. In: INISTA, IEEE International Symposium on., DOI: 10.1109/INISTA.2013.6577668 (2013).

16 Asif, M.: Are QM models aligned with Industry 4.0? A perspective on current practices. Journal of Cleaner Production 258, 120820 (2020).

17 Strickland, Jeffrey - Predictive analytics using R (2016). Lulu.com, ISBN-10: 131284101X; ISBN-13: 978-1312841017.

18 Machine learning for predictive maintenance: where to start? https://medium.com/bigdatarepublic/machine-learning-for-predictive-maintenancewhere-to-start-5f3b7586acfb [Link accessible as July 3 2020].

19 Perera, S., Alwis. R.: Machine Learning Techniques for Predictive Maintenance, https://www.infoq.com/articles/machine-learning-techniques-predictivemaintenance/ [Link accessible as June 29 2020].

20 Coombs, Crispin \& Hislop, Donald \& Barnard, Sarah \& Taneva, Stanimira. (2017). Impact of artificial intelligence, robotics and automation technologies on work rapid evidence review. 10.13140/RG.2.2.32405.42727.

21 Marinov, M., Lazarov, V.: Intuitionistic fuzzy robot motion control. Problems of Engineering Cybernetics and Robotics 69, 40-51 (2018).

22 Pleva, M., Ondas, S.: Speech applications for human - robot interaction systems. Problems of Engineering Cybernetics and Robotics 69, 3-14 (2018).

23 Huang, B.: It's Here! How AI Robot Will Revolutionize Manufacturing. https://towardsdatascience.com/its-here-how-ai-robot-will-revolutionizemanufacturing-44ce784438d4 [Link accessible as June 26 2020].

24 Mihajlovic, I.: Everything You Ever Wanted to Know About Computer Vision. https://towardsdatascience.com/everything-you-ever-wanted-to-know-aboutcomputer-vision-heres-a-look-why-it-s-so-awesome-e8a58dfb641e [Link accessed July 3 2020].

25 Venables, M.: An Overview of Computer Vision. https://towardsdatascience.com/an-overview-of-computer-vision-1f75c2ab1b66 [Link accessed July 3 2020].

26 Kakani, V., Nguyen, V.H., Kumar, B.P., Kim, H., \& Pasupuleti, V.R. (2020). A critical review on computer vision and artificial intelligence in food industry. 
27 Using Artificial Intelligence in Inventory Management. https://clearspider.net/blog/using-artificial-intelligence-in-inventory-management [Link accessed June 28 2020].

28 Global artificial intelligence industry whitepaper, https://www2.deloitte.com/content/dam/Deloitte/cn/Documents/technology-mediatelecommunications/deloitte-cn-tmt-ai-report-en-190927.pdf [Link accessible as July 3 2020].

29 Haarman, M., Mulders, M., Vassiliadis, C.: 2017. Predictive Maintenance 4.0 Predict The Unpredictable. 1st ed. [ebook] Amsterdam: Pricewaterhouse Coopers B.V. https://www.pwc.nl/nl/assets/documents/pwc-predictive-maintenance-4-0.pdf [Accessed June 8 2020].

30 Mustakerov, I., Borissova, D.: Modular systems design via multi-objective optimization. AMO - Advanced Modeling and Optimization, 15(2), 421-430 (2013).

31 Predictive Maintenance: What is it \& What are the Benefits?, https://www.onupkeep.com/learning/maintenance-types/predictive-maintenance [Link accessed July 3 2020]. 